\title{
Mechanism of the initial stages of nitrogen-doped single-walled carbon nanotube growth
}

\author{
Toma Susi, ${ }^{* a}$ Giorgio Lanzani $^{b, c}$, Albert G. Nasibulin ${ }^{a}$, Paola Ayala ${ }^{d}$, Tao Jiang ${ }^{e}$, \\ Thomas Bligaard ${ }^{e}$, Kari Laasonen ${ }^{\mathrm{c}, \mathrm{f}}$, and Esko I. Kauppinen $* a$ \\ ${ }_{5}$ Received (in $\left.X X X, X X X\right) X t h X X X X X X X X X 20 X X$, Accepted Xth $X X X X X X X X X 20 X X$ \\ DOI: $10.1039 / b 000000 x$
}

We have studied the mechanism of the initial stages of nitrogen-doped single-walled carbon nanotube growth illustrated for the case of a floating catalyst chemical vapor deposition system, which uses carbon monoxide $(\mathrm{CO})$ and ammonia $\left(\mathrm{NH}_{3}\right)$ as precursors and iron as catalyst. We performed first-principles

10 electronic-structure calculations, fully incorporating the effects of spin polarization and magnetic moments, to investigate the bonding and chemistry of $\mathrm{CO}, \mathrm{NH}_{3}$, and their fragments on a model $\mathrm{Fe}_{55}$ icosahedral cluster. A possible dissociation path for $\mathrm{NH}_{3}$ to atomic nitrogen and hydrogen was identified, with a reaction barrier consistent with an experimentally determined value we measured by tandem infrared and mass spectrometry. Both $\mathrm{C}-\mathrm{C}$ and $\mathrm{C}-\mathrm{N}$ bond formation reactions were found to be barrierless 15 and exothermic, while a parasitic reaction of $\mathrm{HCN}$ formation had a barrier of over $1 \mathrm{eV}$.

\section{Introduction}

Substitutional doping with nitrogen atoms $\mathrm{s}^{1-3}$ has been proposed as a possible way for controlling the electronic properties of carbon nanotubes (CNTs). A variety of methods ${ }^{4}$ are available for the 20 synthesis of nitrogen-doped multiwalled carbon nanotubes $(\mathrm{N}$ MWCNTs $)^{5}$. However, synthesis of single-walled nitrogen-doped carbon nanotubes (N-SWCNTs) ${ }^{2}$ has struggled to overcome difficulties foreign to the synthesis of pristine material, which is nowadays produced clean in gram quantities. Efforts to master 25 the synthesis of N-SWCNTs have been hindered by an acute lack of knowledge about the chemistry involved and the growth mechanism ${ }^{2,6,7}$, although it should also be noted that despite intensive studies, the mechanism of even pristine CNT growth is still controversial.

30 Furthermore, although N-MWCNTs have been widely studied, the correlation between their morphology, crystallinity and properties is not completely understood. The base growth

\footnotetext{
${ }^{a}$ NanoMaterials Group, Department of Applied Physics, Aalto University School of Science, P.O. Box 15100, FI-00076 Aalto, Espoo, Finland

${ }^{b}$ Thule Institute, University of Oulu, P.O. Box 7300, FI-90014 Oulu, Finland

${ }^{c}$ Department of Chemistry, University of Oulu, P.O. Box 3000, FI-90014 Oulu, Finland

${ }^{d}$ University of Vienna, Faculty of Physics, Strudlhofgasse 4, 1090 Wien, Austria,

${ }^{e}$ CAMd, Technical University of Denmark, Building 307, 2800 Kongens Lyngby, Denmark

${ }^{f}$ Department of Chemistry, Aalto University School of Science, P.O. Box 16100, FI-00076 Aalto, Espoo, Finland

$\dagger$ Electronic Supplementary Information (ESI) available: details of the calculations of absorption geometries and of the HCN formation barrier. See DOI: $10.1039 / \mathrm{b} 000000 \mathrm{x} /$
}

mechanism is commonly proposed to be responsible for the characteristic bamboo-shaped structure ${ }^{8,9}$. This model assumes 35 that the catalyst particles dissociate reactant molecules into $\mathrm{N}$ and $\mathrm{C}$ atoms, whose incorporation into or onto the catalyst nanoparticle results in the walls being pushed away to form a tubular structure. However, no studies have directly addressed the mechanism by which the precursors decompose ${ }^{6}$. The role of 40 nitrogen in N-MWCNTs formation is suggested to be related to the generation of pentagons along with hexagons ${ }^{10}$, because it is believed that the presence of $\mathrm{N}$ in the carbon deposit results in surface strain, leading to a 'pulsed' effect in which the $\mathrm{C} / \mathrm{N}$ surface atoms detach from the metal particle intermittently, ${ }_{45}$ leading to the compartmentalized structure observed ${ }^{11}$. However, it should be noted that this model cannot be applied to $\mathrm{N}$ SWCNTs, which obviously cannot exhibit compartmentalized (bamboo-like) structures.

To our knowledge, there is only one tentative model proposed ${ }_{50}$ for N-SWCNT growth ${ }^{12}$, which further does not discuss the very first stages of growth. Addressing the mechanism of chemical reactions that yield atomic carbon and nitrogen on the catalytic particle $^{13}$ is a crucial first step towards understanding the CVD synthesis of N-SWCNTs. While a decade ago most theoretical 55 studies tried to describe surfaces either on a qualitative level using empirical parameters or invoked rather severe approximate models, there is now a large class of surface system that can be addressed quantitatively based on first principles electronic structure calculation methods ${ }^{14,15}$. This progress is mainly due to 60 advances in computer power and the development of efficient algorithms. However, only small precursor molecules can be treated at this level of accuracy, while as noted by Ewels et al. ${ }^{6}$, most N-CNT synthesis methods use complex molecules as their carbon and nitrogen feedstocks. 


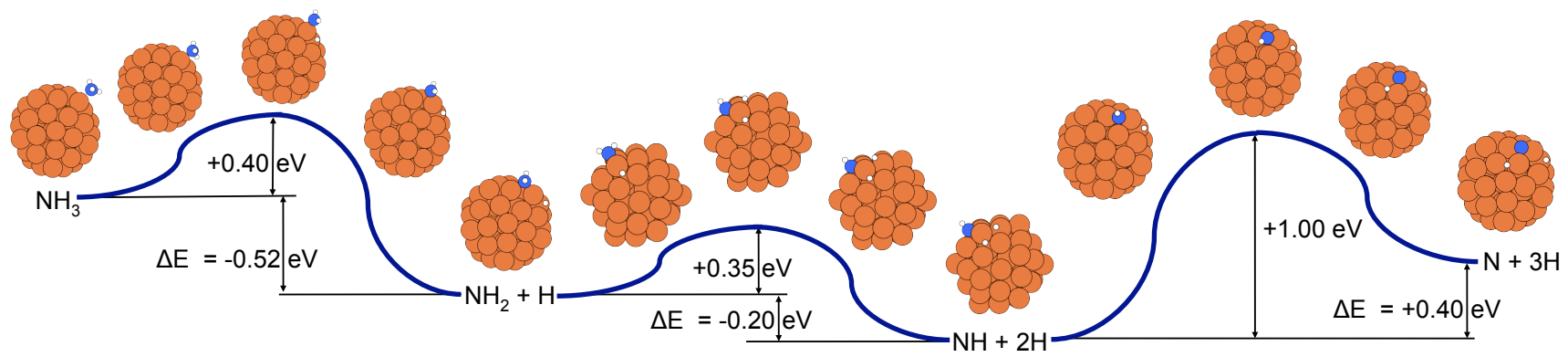

Figure 1. Calculated activation barriers and reaction energies for each step of $\mathrm{NH}_{3}$ decomposition $\left(\mathrm{NH}_{3} \rightarrow \mathrm{NH}_{2}+\mathrm{H} \rightarrow \mathrm{NH}+2 \mathrm{H} \rightarrow \mathrm{N}+3 \mathrm{H}\right)$. The large spheres are $\mathrm{Fe}$ atoms (orange in the web), while $\mathrm{N}$ are medium (blue), and $\mathrm{H}$ small (white).

Recently, the synthesis of N-SWCNTs was shown ${ }^{16}$ to be feasible using a floating catalyst reactor with carbon monoxide (CO) and ammonia $\left(\mathrm{NH}_{3}\right)$, with iron as catalyst. This combination of two small precursor molecules, each with only a 5 single $\mathrm{C}$ or $\mathrm{N}$ atom, presents the simplest possible chemistry. Furthermore, in a floating catalyst approach, the particles interact with precursor molecules in the gas phase, so both the tip and base growth mechanisms are equivalent.

\section{Methods}

10 To study the growth mechanism of N-SWCNTs motivated by the experimental system mentioned above ${ }^{16}$, we performed first principles calculations to understand the chemistry of the precursor reactions on a model $\mathrm{Fe}_{55}$ catalyst cluster ${ }^{14,15}$. The target of the modeling was the critical very first stages of N${ }_{15} \mathrm{SWCNT}$ growth. We believe that the adsorbed $\mathrm{CO}$ and $\mathrm{NH}_{3}$ first dissociate into surface atomic $\mathrm{C}$ and $\mathrm{N}$,

$$
\begin{array}{ll}
\text { and } & \mathrm{CO}_{\text {ads }}-\mathrm{C}_{\text {ads }}+\mathrm{O}_{\text {ads }} \\
20 & \mathrm{NH}_{3 \mathrm{ads}}-\mathrm{N}_{\mathrm{ads}}+3 \mathrm{H}_{\text {ads }} .
\end{array}
$$

Since there is more carbon than nitrogen available on the cluster, the more numerous carbon atoms then react with each other and the nitrogen atoms to form the hexagonal carbon lattice 25 with nitrogen dopants, starting from

and

$$
\mathrm{C}_{\mathrm{ads}}+\mathrm{C}_{\mathrm{ads}} \rightarrow \mathrm{C}-\mathrm{C}_{\mathrm{ads}}
$$

$$
\mathrm{C}_{\mathrm{ads}}+\mathrm{N}_{\mathrm{ads}} \rightarrow \mathrm{C}-\mathrm{N}_{\mathrm{ads}} \text {, }
$$

while the remaining $\mathrm{O}$ atom reacts with additional $\mathrm{CO}$ to form $\mathrm{CO}_{2}$ according to the well-known Boudouard reaction ${ }^{15,17}$. The reactions above seem simple, but in reality the catalyst surface has a high coverage of the molecular precursors and their 35 fragments, so there are complicated atom-atom interactions.

We concentrated our attention on the simplest $\mathrm{CO}$ and $\mathrm{NH}_{3}$ reactivity and characterized the stable stationary points of the species involved in the reactions mentioned above by means of density functional theory (DFT). For the electronic structure 40 calculations we used DFT with gradient corrections (GGA) for the exchange and correlation (RPBE functional ${ }^{18}$ ). The calculations were done with the GPAW code ${ }^{19}$, with only the valence electrons considered explicitly. Iron has a large magnetic moment, and in the cluster geometry, the spin orientation is
${ }_{45}$ subtle. Therefore, we fully incorporated the effects of spin polarization and collinear magnetic moments in our calculations ${ }^{14}$. The cluster geometry was fully relaxed and free to reconstruct in the presence of adsorbates. Although the calculations were performed at zero temperature, a smearing so temperature was used as a convergence parameter in the minimization of the Kohn-Sham energy. The adequacy of the computational parameters has been tested against the calculation of cluster geometry. The method has been detailed in our previous publications ${ }^{14,15}$.

55 To improve the linking of the simulations to experiments, the reaction barriers extracted from the simulations are compared with experiental values measured using Fourier transform infrared (FTIR) spectroscopy and residual gas analysis (RGA) via mass spectrometry in tandem. Gas-phase thermal decomposition 60 of $\mathrm{NH}_{3}$ over iron nanoparticles deposited on the walls of the reactor used for N-SWCNT synthesis ${ }^{16}$ was investigated in the temperature range of $590-1090{ }^{\circ} \mathrm{C}$. Concentrations of ammonia and water as a function of reactor maximum wall temperature were measured using FTIR (Gasmet DX-4000), and hydrogen ${ }_{65}$ and nitrogen using RGA (MKS Cirrus). The inlet gas consisted of $800 \mathrm{~cm}^{3} / \mathrm{min}$ of $\mathrm{Ar}$ along with $500 \mathrm{ppm}$ of $\mathrm{NH}_{3}$.

\section{Results}

As a first step, we obtained information about the stability of $\mathrm{CO}$, $\mathrm{NH}_{3}$ and their fragments on the $\mathrm{Fe}_{55}$ cluster (comparable to earlier 70 results obtained with the VASP $\operatorname{code}^{14,20}$ ). As the second step, we considered the reaction barriers on the surface. Studying the reaction barriers is more demanding, and special transition-state search algorithms, like climbing-image nudged elastic band (CI$\mathrm{NEB})^{21,22}$, were used for their determination, as detailed in 75 previous publications ${ }^{14,15}$.

Based on CO adsorption and $\mathrm{C}+\mathrm{O}$ co-adsorption data (ESI), a possible dissociation path for $\mathrm{CO}$ was mapped out with the NEB method. The smallest activation energy $(0.50 \mathrm{eV})$ was obtained starting from the optimal adsorption geometry with the $\mathrm{CO}$ 80 molecule perpendicular to the cluster ${ }^{23}(\perp \mathrm{CO})$. This reaction is exothermic by $0.12 \mathrm{eV}$. The barrier has been previously measured to be similar ${ }^{15,24}$, although since the NEB method does not consider entropic effects, a direct comparison is not justified. It should also be noted that this barrier is closer to the experimental 85 results than the previous barrier derived from VASP calculations"s.

Correspondingly, based on $\mathrm{NH}_{3-\mathrm{x}}+\mathrm{xH}(\mathrm{x}=0,1,2,3)$ coadsorption data (ESI), a possible dissociation path for $\mathrm{NH}_{3}$ was 

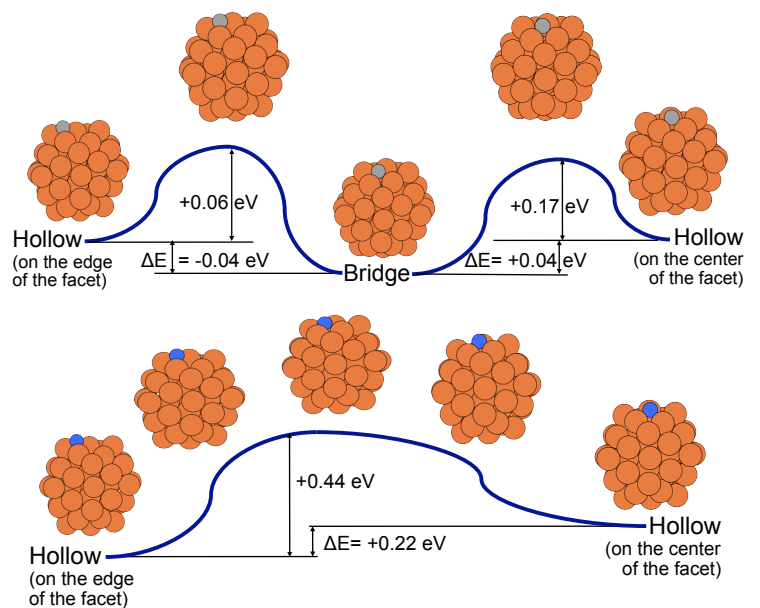

Fig. 2 Proposed reaction paths for the mobility of atomic C (top, grey sphere) and $\mathrm{N}$ (bottom, blue sphere on the web) on the $\mathrm{Fe}_{55}$ nanoparticle. The atomic $\mathrm{C}$ has a possible metastable state between the two stable hollow sites.

calculated with the NEB method. The obtained reaction energy is $-0.32 \mathrm{eV} /$ molecule with an overall barrier of $1.00 \mathrm{eV}$ (Fig. 1). The complete dissociation to atomic nitrogen and hydrogen involves three steps: (1) $\mathrm{NH}_{3} \rightarrow \mathrm{NH}_{2}+\mathrm{H}$; (2) $\mathrm{NH}_{2}+\mathrm{H} \rightarrow \mathrm{NH}+2 \mathrm{H}$; (3) ${ }_{5} \mathrm{NH}+2 \mathrm{H} \rightarrow \mathrm{N}+3 \mathrm{H}$. The rate limiting step is the dissociation of the last hydrogen. The $\mathrm{NH}$ fragment is strongly bound to the surface and the metal-N interaction forces the $\mathrm{NH}$ bond to be perpendicular at the surface.

Once atomic carbon and nitrogen are present on the cluster, it 10 is vital to study their mobility as a preliminary stage of nanotube nucleation. The NEB method was used to study the mobility of carbon $^{23}$ and nitrogen (Fig. 2) on the cluster, and the $\mathrm{C}-\mathrm{C} / \mathrm{C}-\mathrm{N}$ bond forming reactions. The mobility barriers are quite small ( $0.16 \mathrm{eV}$ for $\mathrm{C}$ and $0.40 \mathrm{eV}$ for $\mathrm{N})$, but what is more remarkable, 15 both the $\mathrm{C}-\mathrm{C}$ and the $\mathrm{C}-\mathrm{N}$ bond formation reactions are barrierless and exothermic.

Since there is hydrogen present in the system, the simplest possible further reaction is the formation of hydrogen cyanide $(\mathrm{H}-\mathrm{C} \equiv \mathrm{N})$,

20

$$
\mathrm{CN}_{\mathrm{ads}}+\mathrm{H}_{\mathrm{ads}} \rightarrow \mathrm{HCN}_{\mathrm{ads}},
$$

which has been observed experimentally ${ }^{24}$. This is a parasite reaction, since the $\mathrm{HCN}$ molecule would take away one nitrogen 25 atom otherwise available for doping the carbon network. Based on $\mathrm{CN}$ and $\mathrm{H}$ co-adsorption data, a possible formation path for HCN was calculated with the NEB method. The obtained reaction barrier is $1.65 \mathrm{eV}$ (ESI Fig. S9).

Gas measurements of $\mathrm{NH}_{3}$ decomposition can be found in

30 Figure 3. The accuracy of each concentration measurement was determined from 10 consecutive measurements to be $<10 \mathrm{ppm}$. Three regions can be discerned: (I) a low temperature region (590-740 ${ }^{\circ} \mathrm{C}$ ), where $\mathrm{NH}_{3}$ decomposition is fast and $\mathrm{H}_{2} \mathrm{O}$ is the main product. Oxygen concentration in the reactor from the $\mathrm{Ar}$ 35 carrier gas impurities and environmental leakage was measured to be about $150 \mathrm{ppm}$; (II) an intermediate region $\left(740-940{ }^{\circ} \mathrm{C}\right.$ ), where any additional decomposition of $\mathrm{NH}_{3}$ is into $\mathrm{N}_{2}$ and $\mathrm{H}_{2}$. We believe this corresponds to the catalytic decomposition of $\mathrm{NH}_{3}$ modeled in the simulations; (III) a high temperature region

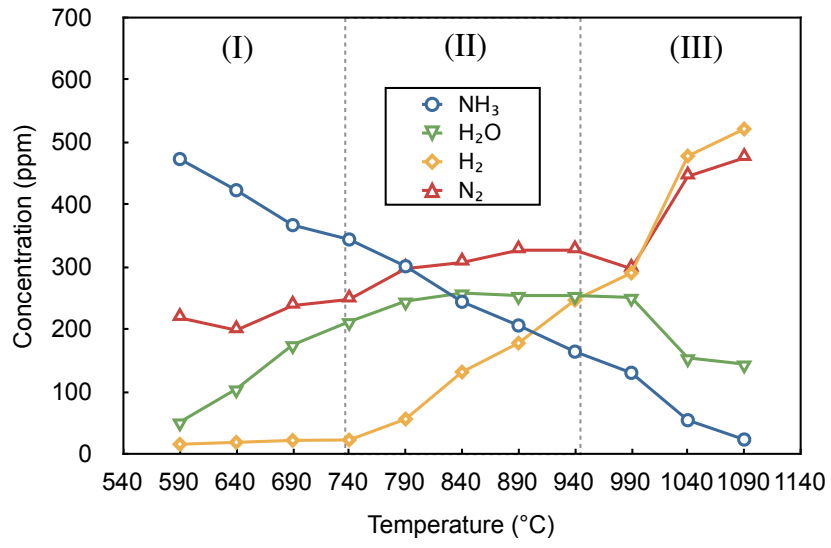

Fig. 3 Concentrations of ammonia and water measured using FTIR, and hydrogen and nitrogen measured using mass spectrometry, as a function of reactor maximum wall temperature.

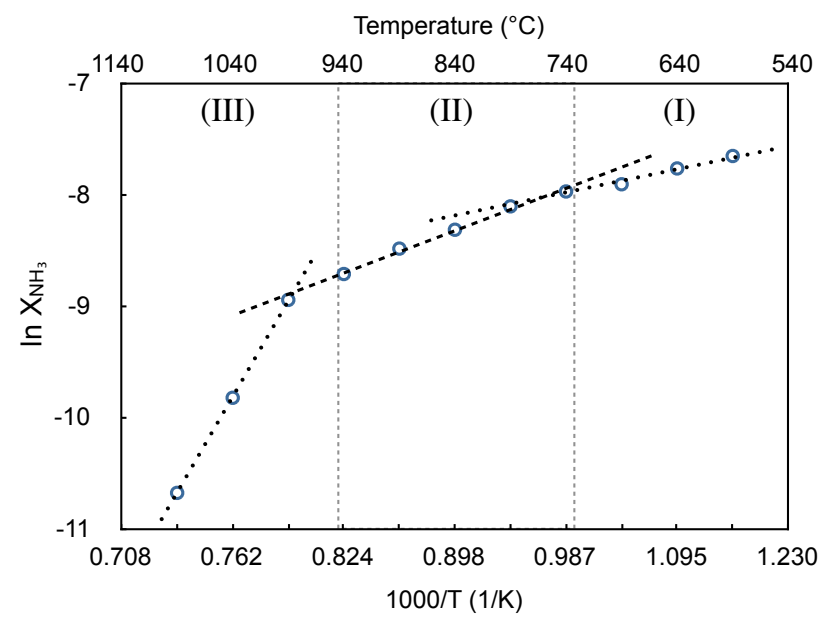

Fig. 4 Arrhenius plot of the mole fraction of $\mathrm{NH}_{3}$, showing three distinct temperature regions (Fig. 3). The dashed and dotted lines are linear fits to the data in the different regions.

$40\left(990-1090{ }^{\circ} \mathrm{C}\right)$, possibly relevant for $\mathrm{NH}_{3}$ self-decomposition in the gas phase. An Arrhenius plot of the mole fraction of $\mathrm{NH}_{3}$ shows the same regions (Fig. 4). Linear least squares fits yield slopes of $28867 \pm 241 \mathrm{~K}, 4978 \pm 190 \mathrm{~K}$ and $1932 \pm 134 \mathrm{~K}$, for the high, middle and low temperature region. These correspond to 45 energies of $-2.49 \pm 0.02 \mathrm{eV},-0.43 \pm 0.02 \mathrm{eV}$ and $-0.17 \pm 0.01 \mathrm{eV}$, respectively. Please note that because the decrease of the mole fraction of $\mathrm{NH}_{3}$ is relevant for the decomposition reaction, the actual barriers come out with the signs reversed.

Similar measurements for the formation of $\mathrm{HCN}$ were 50 performed in the same reactor ${ }^{16}$ using FTIR with 1000 ppm of $\mathrm{NH}_{3}$ in a flow of $400 \mathrm{~cm}^{3} / \mathrm{min} \mathrm{CO}$. Analysis of the Arrhenius plot (ESI Fig. S10) yields a slope of $-15689.7 \mathrm{~K}$, giving an activation energy of $1.35 \mathrm{eV}$. This is consistent with the calculated value $(1.65 \mathrm{eV})$.

\section{${ }_{55}$ 4. Discussion}

The calculated barrier for $\mathrm{NH}_{3}$ decomposition $(1.0 \mathrm{eV})$ differs significantly from the measured one $(0.43 \mathrm{eV})$. The small cluster used for the simulation presents high symmetry and limited size of the facet on which the reaction is constrained. Furthermore, it 
is also possible that the rate-limiting barrier for the cleaving of the final hydrogen would be slight lower if the calculation would be performed on a bigger, irregular cluster. Unfortunately the actual computational capability restricts the size of the simulated 5 cluster to the one here used. The nitrogen in $\mathrm{NH}$ is tetrahedrally $\mathrm{sp}^{3}$ coordinated and the final $\mathrm{H}$ is pointed away from the surface, making it more difficult to reach the surface. This is an artifact of the model cluster and most likely not relevant for a real particle, where a step or a defect would lower this barrier. In the reactive 10 conditions at high temperature in the reactor, the suspended nanoparticle could also be at least in part melted, which we could not take into account in the simulations directly. Finally, considering that the theoretical and experimental data are not directly comparable because of the inability of the NEB results to 15 incorporate entropy change, the simulations can be said to be consistent with the experiments.

Alternatives to the $\mathrm{HCN}$ formation reaction pathway we identified can be considered. The pathway $\mathrm{HN}+\mathrm{C}$ is not relevant for direct hydrogen cyanide formation ${ }^{25,26}$, since the hydrogen in ${ }_{20} \mathrm{HCN}$ is bonded to the carbon atom, not to the nitrogen (as in hydrogen isocyanide, HNC). Our FTIR measurements specifically detected the formation of $\mathrm{HCN}$, and not $\mathrm{HNC}$. Furthermore, although a formation path $\mathrm{HC}+\mathrm{N}$ is common for $\mathrm{HCN}$ formation from hydrocarbon sources ${ }^{25,26}$, we do not 25 consider it to be likely since no $\mathrm{CH}$ fragments are present in the precursors. Of course, the contribution of some multi-step pathways cannot be ruled out by the present work.

Thermodynamically, HCN could also be formed ${ }^{27,28}$ in a gas phase reaction between $\mathrm{CO}$ and $\mathrm{NH}_{3}$. However, the formation of ${ }_{30} \mathrm{CH}_{4}, \mathrm{~N}_{2}$ and $\mathrm{H}_{2} \mathrm{O}$ is the most favorable reaction ${ }^{28}$. Although the formation of $\mathrm{HCN}$ is possible in the presence of $\mathrm{H}_{2}$, that reaction should produce $\mathrm{CH}_{4}$ as well ${ }^{28}$, which we do not observe. We also note that the gas phase formation of $\mathrm{HCN}$ is typically not observed in non-hydrocarbon $\left(\mathrm{CO}+\mathrm{H}_{2}\right)$ flames $^{29}$. In any case, in

35 the presence of a good catalyzer (such as the iron nanoparticles), the assisted reaction path will most likely dominate.

Considering the findings as a whole, we believe that the precursor decomposition reactions happen on the nanocluster itself, not at the open edge of the growing N-SWCNT (as ${ }^{40}$ proposed by Sumpter et al. ${ }^{12}$ ). At least in the case ${ }^{16}$ of $\mathrm{CO}$ and $\mathrm{NH}_{3}$, the calculated reaction barriers strongly suggest that the precursors cannot significantly self-decompose without catalyst at the middle temperature region relevant for nanotube growth. Thus nitrogen is incorporated continuously into the nanotube at 45 the root, not at the leading edge. Cap nucleation and detachment are likely similar to what has been proposed for SWCNTs ${ }^{30,31}$, as are the dynamics of the steady-state growth ${ }^{32-34}$. However, peculiar to the $\mathrm{N}$-doped case, if the nitrogen feedrate is too large, nanotube growth has been found to terminate ${ }^{16}$. This can be either ${ }_{50}$ due to a saturation and breakdown of the hexagonal carbon network by nitrogen, or to nitrogen blocking the adsorption sites on the cluster and preventing the sufficient input of further carbon $^{35}$, slowing down and eventually stopping the growth. Another possibility is that $\mathrm{NH}_{3}$ accelerates the production of 55 active gaseous compounds, which compete with $\mathrm{sp}^{2}$ bond formation (etching effect). If the feedrate is suitable, growth continues in the steady state, with the nanotube being continuously doped with nitrogen until the particle leaves the active zone of the reactor ${ }^{36}$.

60 The next step would be to study of the formation of larger fragments, such as $\mathrm{C}_{2} \mathrm{~N}, \mathrm{C}_{3} \mathrm{~N}$, and beyond. However, this would require larger facets than the $\mathrm{Fe}_{55}$ cluster enables. A larger cluster would in turn require at least a doubling of the number of iron atoms in the simulations, entailing an enormous increase in the 65 computational resources required. Nonetheless, this study marks the first explicit study of the initial stages of N-SWCNT growth. Since no fundamental differences were found in the chemistry of the $\mathrm{C}$ and $\mathrm{N}$ precursors, we believe that the observed difficulties in synthesizing nitrogen-doped SWCNTs can be explained by the 70 fact that N-MWCNTs can tolerate much larger amounts of $\mathrm{N}$ and the defects they entail for the graphitic lattice, while N-SWCNTs are more likely severely disturbed by even relatively small amounts of $\mathrm{N}$ during their nucleation and growth.

\section{Conclusions}

75 The mechanism of the initial stages of nitrogen-doped singlewalled carbon nanotube growth was studied in a system with the simplest possible experimentally feasible chemistry with $\mathrm{CO}$ and $\mathrm{NH}_{3}$ as precursors. The dissociation reaction of $\mathrm{NH}_{3}$ to atomic nitrogen and hydrogen was identified, with a reaction barrier 80 comparable to an experimentally determined value measured by tandem infrared and mass spectrometry. It was found that the chemistry involving $\mathrm{C}$ and $\mathrm{N}$ is rather similar. The formation of a $\mathrm{C}-\mathrm{N}$ bond between atomic carbon and nitrogen was also found to be barrierless and exothermic, while there is a large barrier for 85 parasitic HCN formation. Thus there seems to be no fundamental differences in the mechanism of the precursor reactions.

\section{Acknowledgements}

We acknowledge the generous computational resources provided by CSC (Center for Scientific Computing, Finland). This work ${ }_{90}$ has been supported by the Academy of Finland (project No 128445), Finnish TEKES GROCO Project (1298/31/08), and the Aalto University MIDE-program project CNB-E. P.A. acknowledges an individual Marie Curie Intra-European Fellowship within the 7th European Community Framework 95 Programme.

\section{References}

1 O. Stephan, P. M. Ajayan, C. Colliex, P. Redlich, J. M. Lambert, P. Bernier and P. Lefin, Science, 1994, 266, 1683.

2 P. Ayala, R. Arenal, A. Loiseau, A. Rubio and T. Pichler, Rev. Mod. Phys., 2010, 82, 43.

3 J. Y. Yi and J. Bernholc, Phys. Rev. B, 1993, 47, 1708-1711.

4 C. P. Ewels and M. Glerup, J. Nanosci. Nanotechnol., 2005, 5, 13451363.

5 P. Ayala, R. Arenal, M. H. Rümmeli, A. Rubio and T. Pichler, Carbon, 2010, 48, 585-586.

6 C. P. Ewels, M. Glerup and V. Krstic, in Chemistry of Carbon Nanotubes, eds. V. A. Basiuk and E. V. Basiuk, American Scientific Publishers, 2008

7 R. Arenal and A. Loiseau, B-C-N Nanotubes and Related 110 Nanostructures, 2009, 6, 45-81.

8 M. Terrones, H. Terrones, N. Grobert, W. K. Hsu, Y. Q. Zhu, J. P. Hare, H. W. Kroto, D. R. M. Walton, P. Kohler-Redlich, M. Ruhle, J. P. Zhang and A. K. Cheetham, Appl. Phys. Lett., 1999, 75, 39323934. 
9 K. Suenaga, M. Yudasaka, C. Colliex and S. Iijima, Chem. Phys. Lett., 2000, 316, 365-372.

10 C. H. Lin, H. L. Chang, C. M. Hsu, A. Y. Lo and C. T. Kuo, Diamond and Related Materials, 12, 1851-1857.

511 S. Trasobares, O. Stephan, C. Colliex, W. K. Hsu, H. W. Kroto and D. R. M. Walton, J. Chem. Phys., 2002, 116, 8966-8972.

12 B. G. Sumpter, J. Huang, V. Meunier, J. M. Romo-Herrera, E. CruzSilva, H. Terrones and M. Terrones, Int. J. Quantum Chem., 2009, 109, 97-118.

1013 A. G. Nasibulin, A. Moisala, D. Brown, H. Jiang and E. I. Kauppinen, Chem. Phys. Lett., 2005, 402, 227-232.

14 G. Lanzani, A. G. Nasibulin, K. Laasonen and E. I. Kauppinen, Nano Res., 2009, 2, 660-670.

15 G. Lanzani, A. Nasibulin, K. Laasonen and E. I. Kauppinen, J. Phys.

15 Chem. C, 2009, 113, 12939.

16 T. Susi et al., Chemistry of Materials, 2011, Article ASAP doi:10.1021/cm200111b.

17 A. G. Nasibulin, D. P. Brown, P. Queipo, D. Gonzalez, H. Jiang and E. I. Kauppinen, Chem. Phys. Lett., 2006, 417, 179-184.

2018 B. Hammer, L. Hansen and J. Nørskov, Phys. Rev. B, 1999, 59, 7413.

19 J. Mortensen, L. Hansen and K. Jacobsen, Phys. Rev. B, 2005, 71, 035109.

20 G. Kresse and J. Hafner, J. Phys. Condens. Matter, 1994, 6, 8245

21 G. Henkelman and H. Jónsson, J. Chem. Phys., 2000, 113, 9978.

2522 G. Henkelman, B. Uberuaga and H. Jónsson, J. Chem. Phys., 2000, 113, 9901.

23 G. Lanzani, T. Susi, P. Ayala, A. G. Nasibulin, T. Bligaard, T. Pichler, K. Laasonen and E. I. Kauppinen, Phys. Status Solidi B, 2010, 247 (11-12), 2708-2712.

3024 T. Susi, A. G. Nasibulin, P. Ayala, Y. Tian, Z. Zhu, H. Jiang, C. Roquelet, D. Garrot, J. S. Lauret and E. I. Kauppinen, Phys. Status Solidi B, 2009, 246, 2507-2510.

25 R. M. van Hardeveld, R. A. van Santen and J. W. Niemantsverdriet, J. Phys. Chem. B, 1997, 101, 7901-7907.

3526 S. Delagrange and Y. Schuurman, Cat. Today, 2007, 121, 204-209.

27 G. Schlesinger and S. L. Miller, J. Mol. Evol., 1983, 19, 383-390390.

28 N. Aylward, in Proceedings of the 10th WSEAS International Conference on Mathematical Methods and Computational

40 Techniques in Electrical Engineering, World Scientific and Engineering Academy and Society (WSEAS), Sofia, Bulgaria, 2008, pp. 267-273.

29 T. Takagi, T. Tatsumi and M. Ogasawara, Comb. Flame, 1979, 35, $17-25$.

4530 Y. Ohta, Y. Okamoto, A. Page, S. Irle and K. Morokuma, ACS Nano, $2009,3,8$.

31 M. Moors, H. Amara, T. Visart de Bocarmé, C. Bichara, F. Ducastelle, N. Kruse and J.-C. Charlier, ACS Nano, 2009, 3, 6.

32 Y. Shibuta and S. Maruyama, Chem. Phys. Lett., 2003, 382, 381-386.

5033 S. Irle, Y. Ohta, Y. Okamoto, A. J. Page, Y. Wang and K. Morokuma, Nano Res., 2009, 2, 755-767.

34 A. S. Anisimov, A. G. Nasibulin, H. Jiang, P. Launois, J. Cambedouzou, S. D. Shandakov and E. I. Kauppinen, Carbon, 2010, $48,9$.

5535 A. Harutyunyan, N. Awasthi, A. Jiang, W. Setyawan, E. Mora, T. Tokune, K. Bolton and S. Curtarolo, Phys. Rev. Lett., 2008, 100, 195502.

36 A. G. Nasibulin and S. D. Shandakov, Aerosol Synthesis of SingleWalled Carbon Nanotubes, Wiley-VCH Verlag GmbH \& Co. KGaA,

60 2010. 


\section{Supplementary information}

\section{Mechanism of the initial stages on single-walled nitrogen-doped carbon nanotube growth}

\section{DOI: $10.1039 / \mathrm{c} 1 \mathrm{cp} 20454 \mathrm{~h}$}

Toma Susi ${ }^{1, *}$, Giorgio Lanzani ${ }^{2,3}$, Albert G. Nasibulin ${ }^{1}$, Paola Ayala ${ }^{4}$, Tao Jiang ${ }^{5}$, Thomas Bligaard $^{5}$, Kari Laasonen ${ }^{3,6}$, and Esko I. Kauppinen ${ }^{1, *}$

1 NMG, Department of Applied Physics, Aalto University School of Science and Technology, P.O. Box 15100, FI-00076 Aalto, Espoo, Finland

2 Thule Institute, University of Oulu, P.O. Box 7300, FI-90014, University of Oulu, Finland

3 Department of Chemistry, University of Oulu, P.O. Box 3000, FI-90014, University of Oulu, Finland

4 Fakultät für Physik, Universität Wien, Strudlhofgasse 4, 1090 Wien, Austria,

5 CAMd, Technical University of Denmark, Building 311, 2800 Kongens Lyngby, Denmark

6 Department of Chemistry, Aalto University School of Science and Technology, P.O. Box 16100, FI-00076 Aalto, Espoo, Finland

Email:toma.susi@tkk.fi

Corresponding authors: e-mail toma.susi@tkk.fi and esko.kauppinen@tkk.fi. Phone: +358 50344 1574 (Toma Susi) and +358 405098064 (Esko I. Kauppinen), Fax: +358 94513517 


\section{Supplementary information}

First-principles DFT calculations were used to study $\mathrm{CO}, \mathrm{NH}_{3}, \mathrm{NH}_{2}, \mathrm{NH}$, atomic $\mathrm{C}$ and $\mathrm{N}$ adsorption on icosahedral $\mathrm{Fe}_{55}$ cluster. The geometry optimization was started from the high symmetry adsorption sites of one the 20 triangular face of the cluster. The high symmetry adsorption sites are the top site, octahedral hollow site and tetrahedral bridge sites. In our simulation, the bridge and top site can be in-plane on the cluster face or on its edge/vertices. For

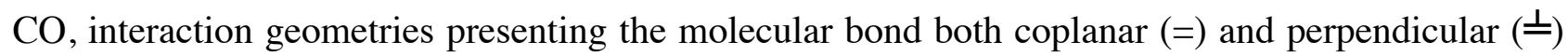
to the cluster surface were considered. When the $\mathrm{CO}$ molecule is placed flat on the cluster, during the geometry optimizations oxygen atoms are not bonding to the surface and the molecule flips up.

Perpendicular adsorption is not favorable when oxygen is toward the surface $(\stackrel{\perp}{=} \mathrm{OC})$. During the relaxations with the molecule adsorbed through the carbon $(\stackrel{\perp}{\perp})$, it spontaneously moves, migrating on the B, D and F sites (Fig. S1) of the surface. To describe quantitatively the energetics of the adsorbed species on the surface, we introduce the interaction energy term (I.E.), defined as

$$
\text { I.E. }=\mathrm{E}_{\mathrm{Fe}_{55} / \mathrm{CO}}-\left(\mathrm{E}_{\mathrm{Fe}_{55}}+\mathrm{E}_{\mathrm{CO}}\right) \text {, }
$$

where $\mathrm{E}_{\mathrm{Fe} 55 / \mathrm{CO}}$ is the total energy of the adsorbate-substrate system, $\mathrm{E}_{\mathrm{Fe} 55}$ of the $\mathrm{Fe}_{55}$ cluster, and $\mathrm{E}_{\mathrm{CO}}$ of the free $\mathrm{CO}$ molecule. I.E. describes the strength of the adsorbate-substrate interaction. In Fig. S1 the I.E. values are reported for the optimized geometries.

The stability of $\mathrm{NH}_{3}$ on the cluster was considered as well. The molecule was set on the vertices, on the edge and on the centre of the icosahedral face of the cluster. For every adsorption site the possible effect of the orientation was considered, though only the interaction N-Fe was favorable, and only the top sites are stable $\left(-0.73 \mathrm{eV}<\right.$ B.E. $\left.\left(\mathrm{NH}_{3}\right)<-0.60 \mathrm{eV}\right)$. The obtained stable geometries and I.E.s (calculated with respect to $\mathrm{E}_{\mathrm{NH} 3}$ ) are reported in Fig. S2.

In order to study $\mathrm{CO}$ and $\mathrm{NH}_{3}$ dissociation with the CI-NEB method, we need to know the preferred adsorption sites for $\mathrm{NH}_{2}, \mathrm{NH}, \mathrm{N}, \mathrm{C}$ and $\mathrm{O}$ atoms on the cluster. Considering $\mathrm{NH}_{2}$, during relaxation the molecule spontaneously moves, migrating to the bridge sites on the facet of the surface $\left(-3.10 \mathrm{eV}<\right.$ B.E. $\left.\left(\mathrm{NH}_{2}\right)<-2.52 \mathrm{eV}\right)$ (Fig. S3). The arrangement presenting the nitrogen 
tetrahedrally coordinated to two hydrogens and two irons atoms is clearly preferred. Similar behavior has been observed considering the interaction of $\mathrm{NH}$ at the high symmetry adsorption site $\left(-4.64 \mathrm{eV}<\right.$ B.E. $\left.\left(\mathrm{NH}_{2}\right)<-3.18 \mathrm{eV}\right)$ (Fig. S4). During geometry optimization, the fragment rearranges, moving to the hollow site, where $\mathrm{N}$ can easily interact with three metal atoms.

Considering atomic carbon and nitrogen, all possible adsorption sites on the surface (Figs. S5 and S6) and below (Figs. S7 and S8) were considered, but only few stable sites for individually adsorbed $\mathrm{C}$ and $\mathrm{N}$ were located. Both of the atoms present similar behavior: the hollow sites on and below the surface are most stable. I.E. values are reported in the figures; for comparisons remember that the reference energy for a non-adsorbed carbon is that of the isolated atom.

Fig. S9 shows a possible formation path for $\mathrm{HCN}$ was calculated with the NEB method. The obtained reaction barrier is $1.65 \mathrm{eV}$. Fig. S10 shows an Arrhenius plot of the mole fraction of HCN as a function of reactor wall temperature. A fit to the linear region yields a slope of $-15689.7 \mathrm{~K}$, which corresponds to an activation energy of $1.35 \mathrm{eV}$.
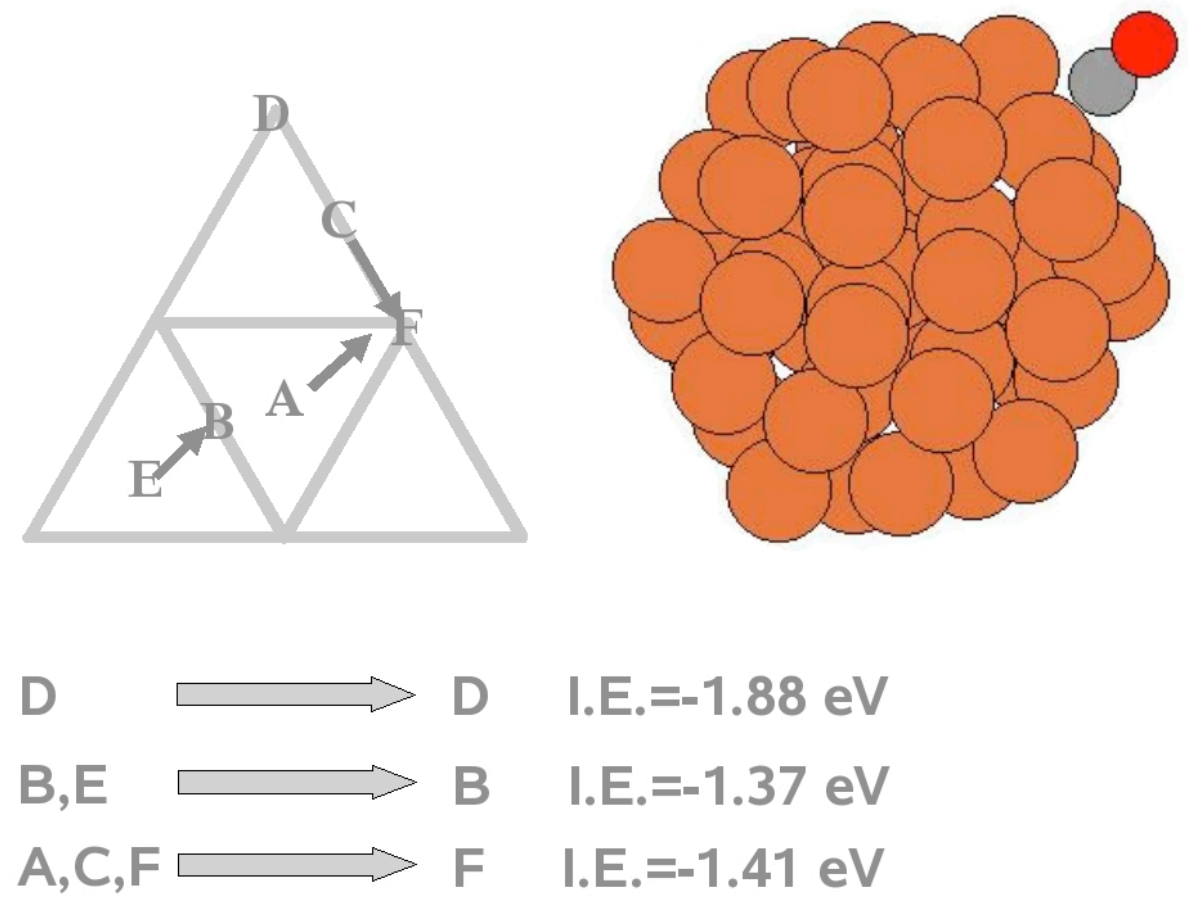

Fig. S1 Behavior observed during geometry relaxation of $\mathrm{CO}$, started from the molecule adsorbed perpendicular at the cluster surface. During optimization, the molecule spontaneously moves to the edge of the facet or to the border of the hollow cavity. 

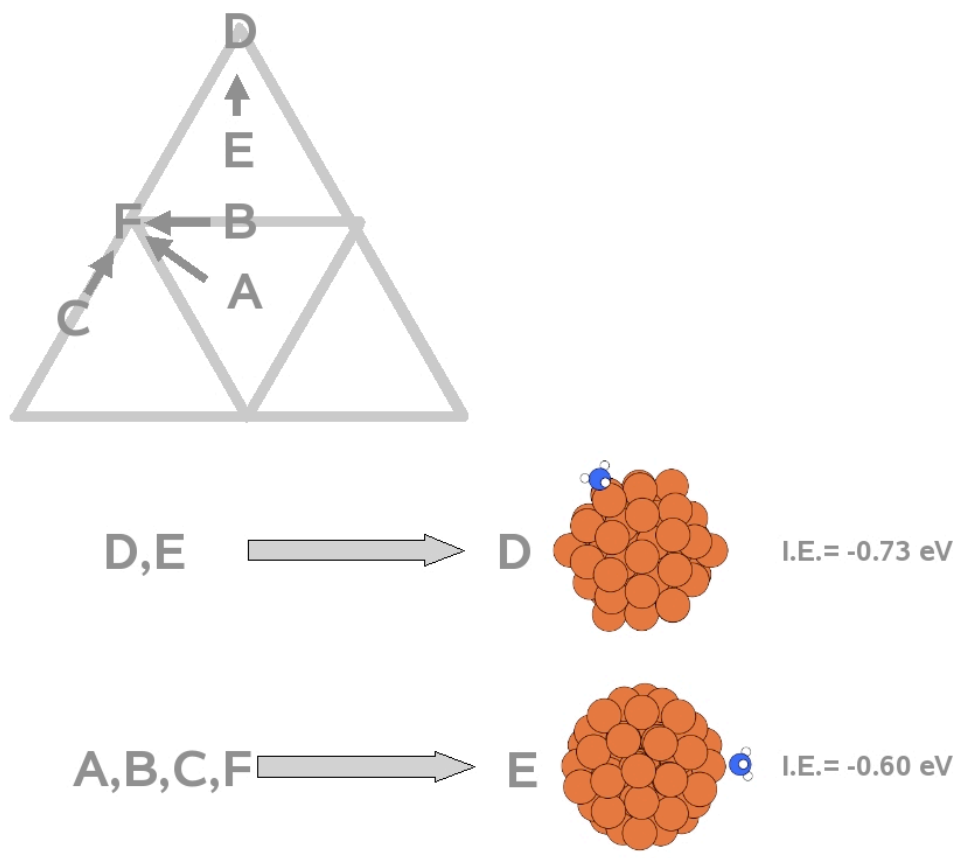

Fig. S2 Behavior observed during geometry relaxation of $\mathrm{NH}_{3}$, started from the molecule adsorbed on the cluster surface. $\mathrm{NH}_{3}$ prefers to interact with the most exposed metal atoms, and after the first $\mathrm{H}$ dissociation, the molecule spontaneously moves to the top sites during geometry optimization.

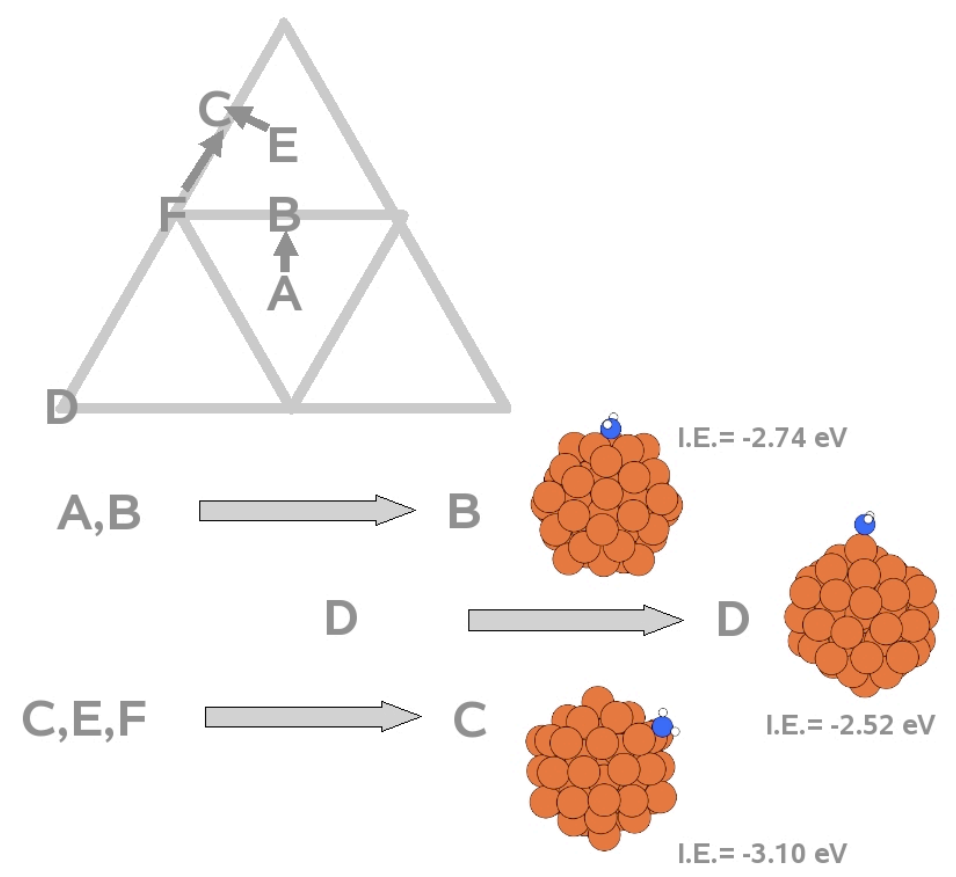

Fig. S3 Behavior observed during geometry relaxation of $\mathrm{NH}_{2}$, started from the molecule adsorbed on the cluster surface. In order to optimize the interaction with the surface and maintain tetrahedral coordination, $\mathrm{NH}_{2}$ spontaneously move to the bridge sites $(\mathrm{C}$ and $\mathrm{B})$ during the optimization. 


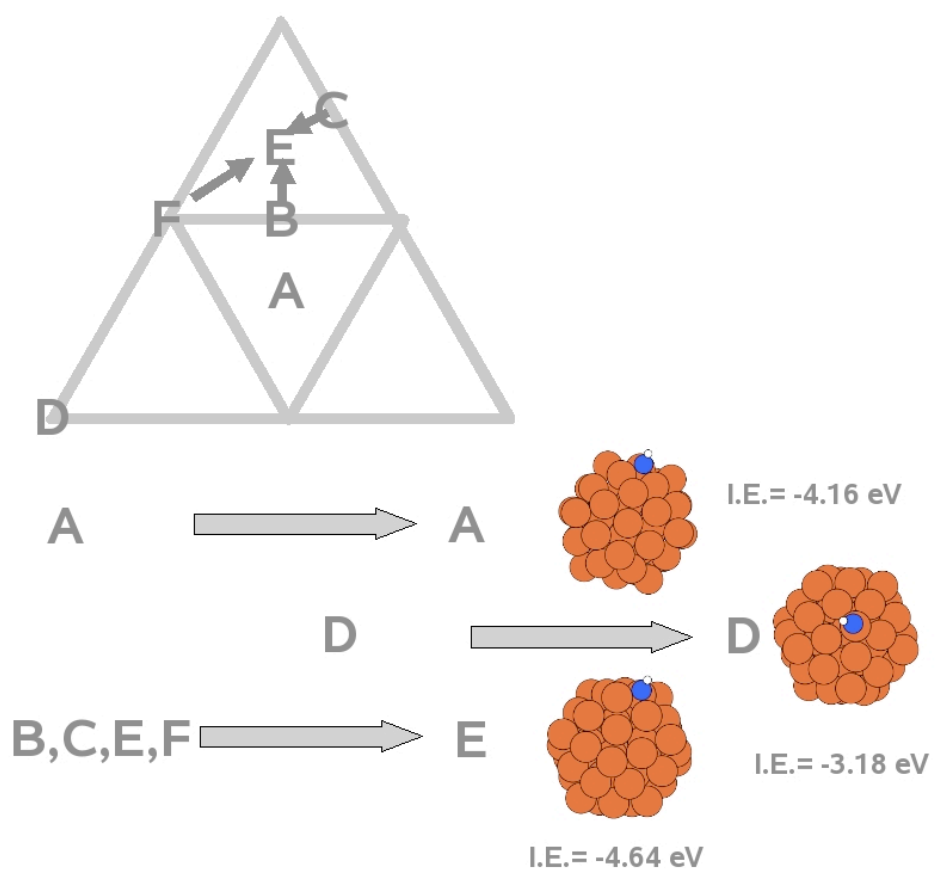

Fig. S4 Behavior observed during geometry relaxation of $\mathrm{NH}$, started from the molecule adsorbed on the cluster surface. In order to optimize interaction with the surface and maintain tetrahedral coordination, $\mathrm{NH}$ spontaneously moves to the hollow sites (A and E) during the optimization.
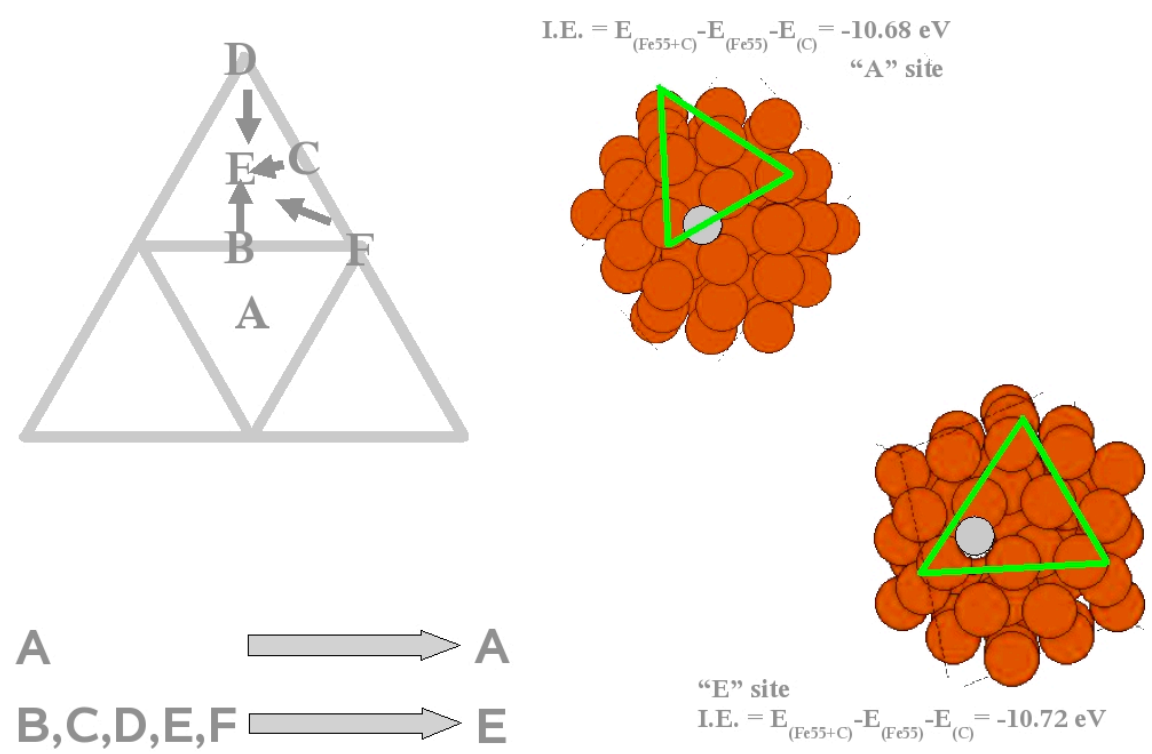

Fig. S5 Behavior observed during geometry relaxation of atomic $\mathrm{C}$ adsorbed on the high symmetry adsorption sites of the cluster surface. The hollow sites (A and E) are the only stable ones, and from the others, the adsorbed $\mathrm{C}$ migrates to the nearest hollow site. 


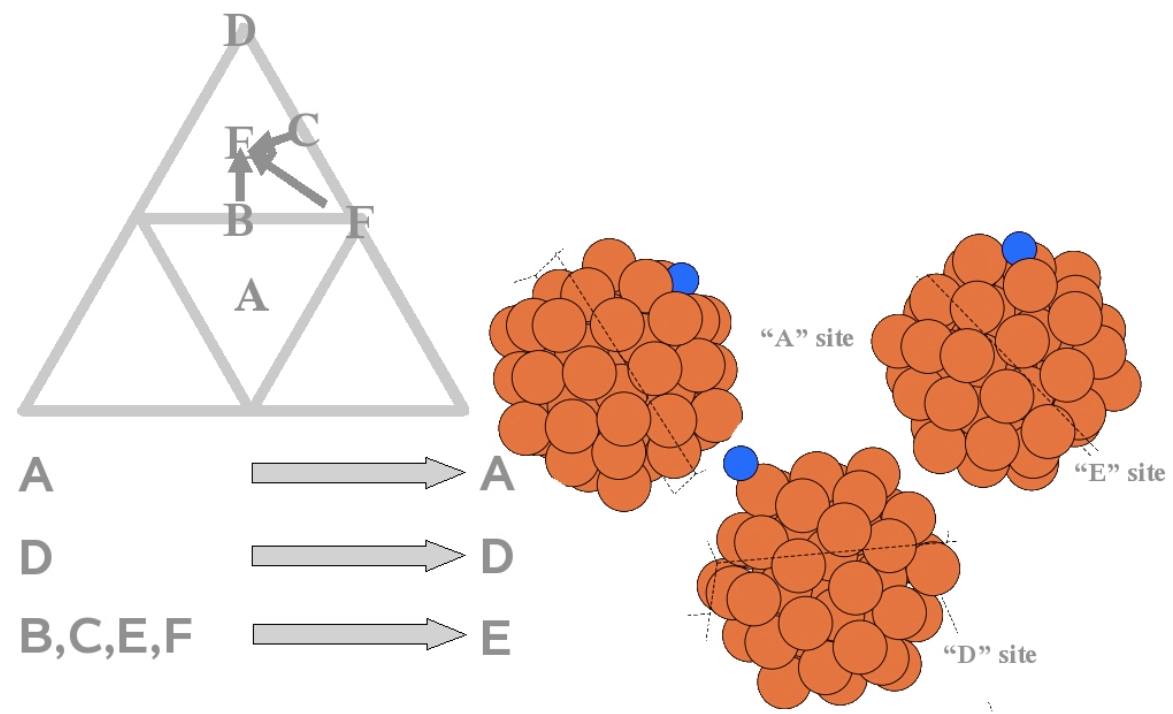

Fig. S6 Behavior observed during geometry relaxation of atomic $\mathrm{N}$ adsorbed on the high symmetry adsorption sites of the cluster surface. The hollow sites (A and E) are the only stable ones, and from the bridge sites, the adsorbed $\mathrm{N}$ migrates on the nearest hollow site.

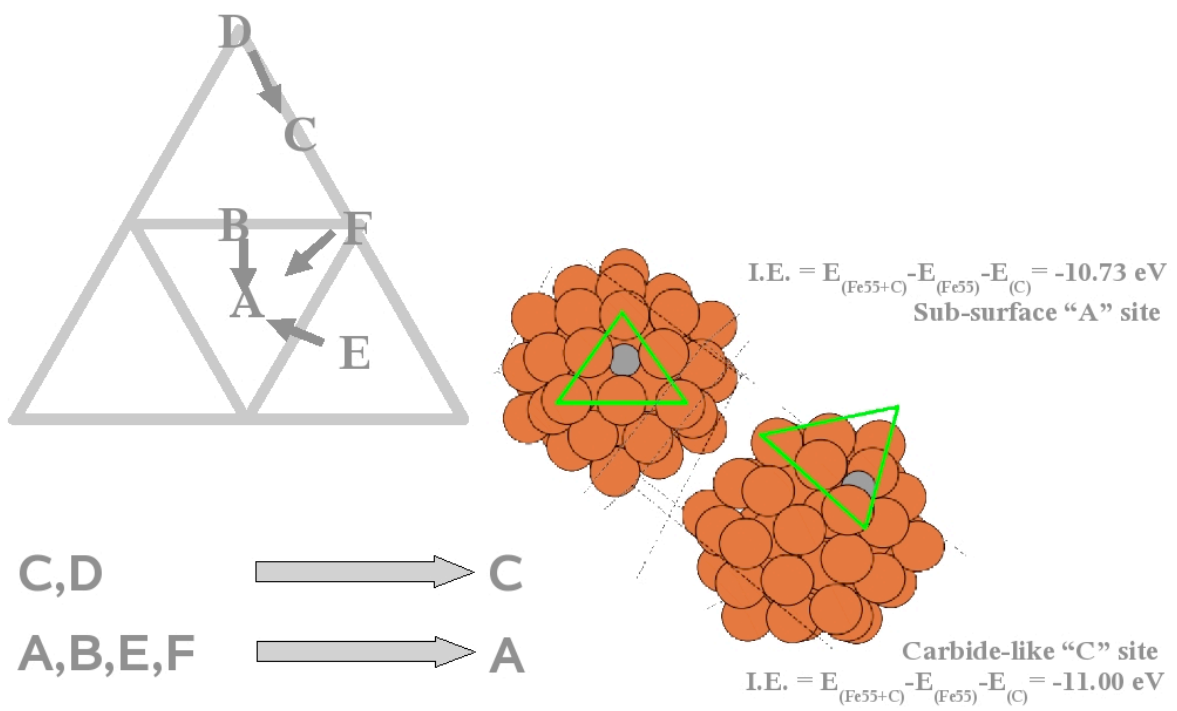

Fig. S7 Behavior observed during geometry relaxation of subsurface atomic $\mathrm{C}$ adsorbed on the high symmetry adsorption sites of the cluster surface. In the majority of cases, the $\mathrm{C}$ atom moves spontaneously to the octahedral cavity below the A site during the geometry optimization. 

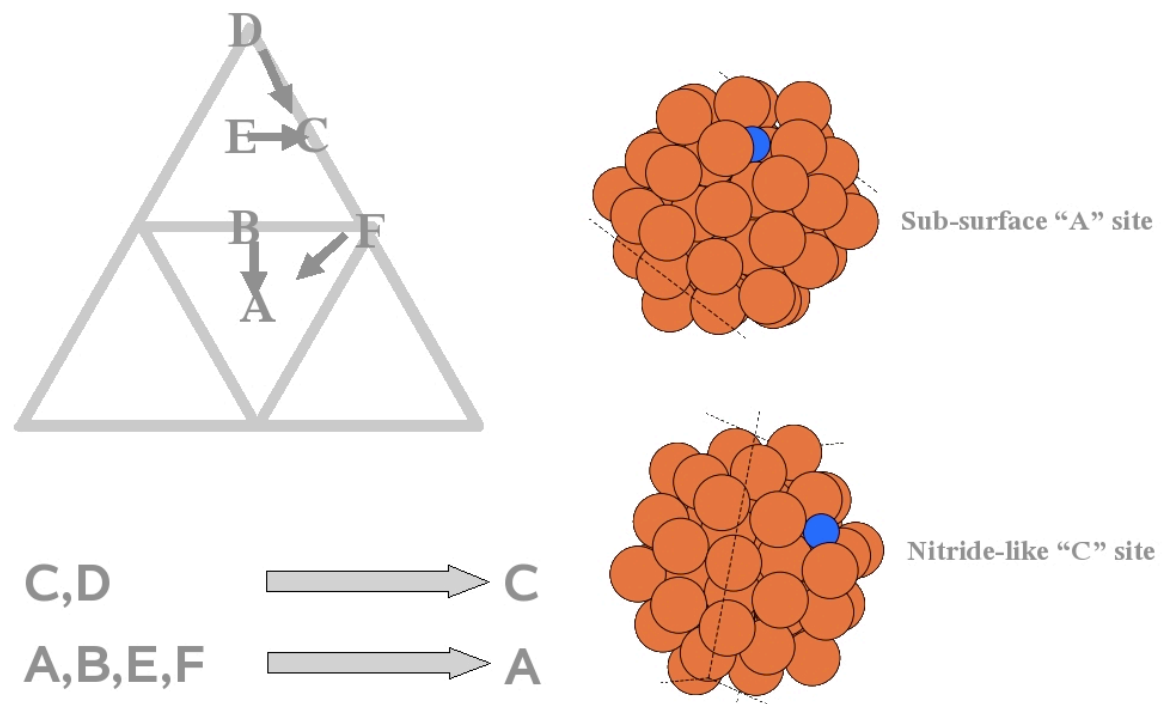

Fig. S8 Behavior observed during geometry relaxation of subsurface atomic $\mathrm{N}$ adsorbed on the high symmetry sites of the cluster surface. The behavior is similar to what is observed for atomic $\mathrm{C}$.

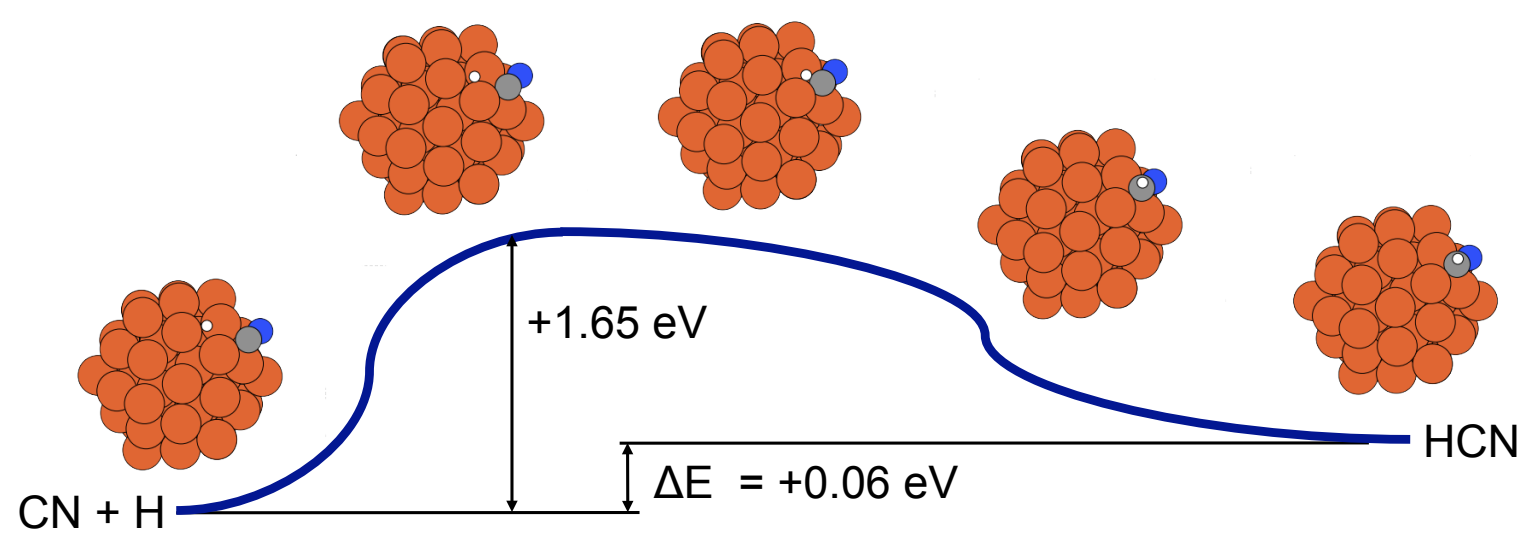

Fig. S9 Proposed reaction path for $\mathrm{HCN}$ formation reaction on the cluster. 


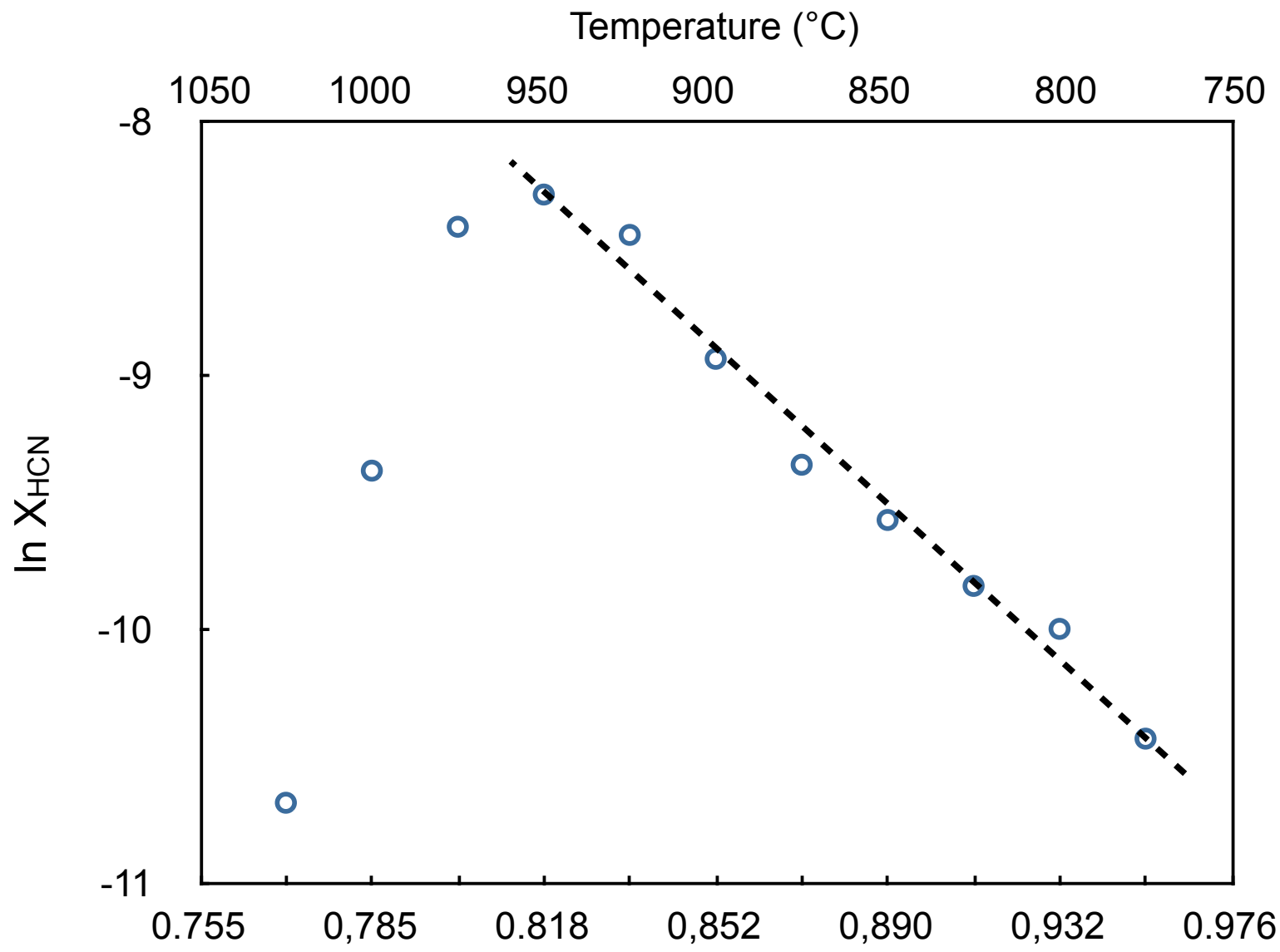

Fig. S10 Arrhenius plot of the mole fraction of HCN measured using FTIR as a function of reactor maximum wall temperature. 